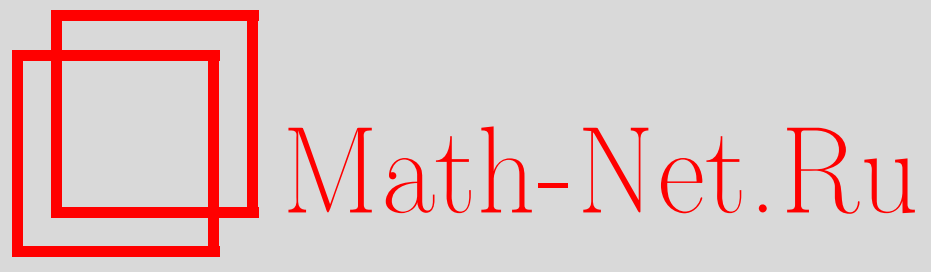

Е. С. Половинкин, Об одном следствии теоремы Шаудера, Матем. заметки, 2014, том 96, выпуск 6, 953-954

DOI: https://doi.org/10.4213/mzm10564

Использование Общероссийского математического портала Math-Net.Ru подразумевает, что вы прочитали и согласны с пользовательским соглашением http://www . mathnet.ru/rus/agreement

Параметры загрузки:

IP: 54.164 .48 .24

26 апреля 2023 г., 09:52:45

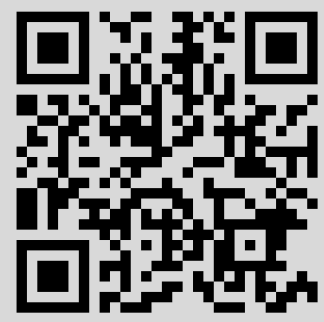




\section{Об одном следствии теоремы Шаудера}

\section{Е. С. Половинкин}

1. Введение. В книге Ли и Маркуса [1] в $§ 4.1$ приведено некоторое "топологическое отступление", являющееся следствием теоремы Брауэра о неподвижной точке. Это следствие при некоторых условиях на непрерывное отображение утверждало о непустоте внутренности образа единичного шара из $\mathbb{R}^{n}$ при таком отображении. Следствие нашло широкое применение в исследованиях этих авторов (см., например, доказательство теоремы 3 в $\left.4_{4.1}[1]\right)$. Также это следствие применялось автором статьи в работах [2], [3]. В данной заметке мы докажем обобщение указанного результата на случай банаховых пространств.

2. Вспомогательный результат. Пусть $E$ - действительное банахово пространство и $B_{r}\left(x_{0}\right)$ - открытый шар радиуса $r>0$ с центром в точке $x_{0}$ в пространстве $E$. Как обычно, обозначим через $\bar{A}, \operatorname{int} A$ и $\partial A$ замыкание, внутренность и границу множества $A \subset E$ соответственно. Нам потребуется следствие теоремы Шаудера (см. [4; гл. 5, п. 10]).

Лемма. Пусть задано замкнутое выпуклое множество $А$ из банахова пространства E. Тогда у любого непрерывного отображения $f: A \rightarrow A, y$ которого множество значений $f(A)$ относительно компактно, существует неподвижная точка.

ДокАЗАТЕЛЬСтво. По условию множество $B \doteq \overline{f(A)}$ является компактным множеством. В свою очередь, замыкание выпуклой оболочки $C \doteq \overline{\operatorname{co}} B$ множества $B$ по теореме Мазура [4; гл. 5, п. 2] является выпуклым компактом в $E$. Так как $f(A) \subset A$, а $A$ выпукло и замкнуто, то и $C \subset A$. В результате $f(C) \subset f(A) \subset C$. Итак, $C$ - выпуклый компакт, $C \subset A$ и $f: C \rightarrow C$. По теореме Шаудера существует неподвижная точка $b \in C \subset A$, т.е. $f(b)=b$ и $b \in A$.

\section{3. Основной результат.}

Теорема. Пусть в банаховом пространстве $Е$ задано ограниченное замкнутое въпуклое множество $A \subset E$ с непустой внутренностью. Пусть отображение $f: A \rightarrow E$ непрерывно, а множество значений $(f-I)(A)$ относительно компактно (здесъ $I(x)=x$ для любого $x \in E)$. Пусть для некоторой точки $y_{0} \in \operatorname{int} A$ справедливы неравенства

$$
\|f(x)-x\| \leqslant\left\|x-y_{0}\right\| \quad \text { для всех } \quad x \in \partial A .
$$

Тогда существует точка $x_{0} \in A$ такая, что $f\left(x_{0}\right)=y_{0}$.

ДокАЗАтЕЛЬСтво. Достаточно рассмотреть случай, когда $y_{0}=0$. В самом деле, проведя сдвиг в пространстве $E$ по формуле $\widetilde{x} \doteq x-y_{0}$ и сделав замены $\widetilde{A} \doteq A-y_{0}, \widetilde{f}(\widetilde{x}) \doteq$ $f(x)-y_{0}$, приводим условия теоремы к случаю, когда $y_{0}=0$.

Итак, полагаем в дальнейшем, что $y_{0}=0 \in \operatorname{int} A$, а условие (1) принимает вид

$$
\|f(x)-x\| \leqslant\|x\| \quad \text { для всех } \quad x \in \partial A .
$$

Так как по условию множество $A$ ограничено и $0 \in \operatorname{int} A$, существуют числа $r, d$ такие, что $0<r<d$ и $\overline{B_{r}(0)} \subset A \subset \overline{B_{d}(0)}$. Для множества $A$ определим функцию Минковского $\mu(x) \doteq$ $\inf \{t>0 \mid x / t \in A\}$. Как известно (см, например, [5], [6]), функция Минковского $\mu(x)$

Работа выполнена при поддержке Российского фонда фундаментальных исследований (грант № 13-01-00295).

DOI: $10.4213 / \mathrm{mzm} 10564$ 
выпукла, полунепрерывна снизу и положительно однородна. В силу отмеченных выше ограничений на множество $A$ она также удовлетворяет неравенствам

$$
\frac{1}{d}\|x\| \leqslant \mu(x) \leqslant \frac{1}{r}\|x\|,
$$

откуда в частности следует, что функция $\mu(x)$ удовлетворяет условию Липшица с константой Липшица $1 / r$.

Определим отображение $g: E \rightarrow A$ по формуле $g(x) \doteq x / \mu(x)$ при $x \notin A$, и $g(x)=x$ при $x \in A$. В силу приведенных свойств функции Минковского, отображение $g$ является непрерывным, $g(E)=A$ и $g(x) \in \partial A$ при $x \notin \operatorname{int} A$.

Определим отображение $v: A \rightarrow A$ по формуле $v(x) \doteq g(x-f(x))$. Очевидно, что это непрерывное отображение и множество значений $v(A)$ относительно компактно. По лемме существует точка $x_{0} \in A$ такая, что $v\left(x_{0}\right)=x_{0}$. Покажем, что $x_{0}-f\left(x_{0}\right) \in A$. Допустим противное, т.е. $x_{0}-f\left(x_{0}\right) \notin A$. Тогда из свойств функции $g$ следует, что $g\left(x_{0}-f\left(x_{0}\right)\right) \in$ $\partial A$, т.е. $x_{0} \in \partial A$. Кроме того, из свойств функции Минковского получаем неравенство $\mu\left(x_{0}-f\left(x_{0}\right)\right)>1$ и равенство $\left(x_{0}-f\left(x_{0}\right)\right) / \mu\left(x_{0}-f\left(x_{0}\right)\right)=x_{0}$, т.е.

$$
\frac{\left\|x_{0}-f\left(x_{0}\right)\right\|}{\left\|x_{0}\right\|}=\mu\left(x_{0}-f\left(x_{0}\right)\right)>1 .
$$

Однако последнее неравенство противоречит условию (2). Таким образом, показали, что $x_{0}-f\left(x_{0}\right) \in A$. В силу этого из определения отображения $g$ и определения точки $x_{0} \in A$ получаем равенство $g\left(x_{0}-f\left(x_{0}\right)\right)=x_{0}-f\left(x_{0}\right)=x_{0}$, т.е. $f\left(x_{0}\right)=0$, что и требовалось доказать.

СледствиЕ. Пусть в банаховом пространстве $E$ отображение $f: \overline{B_{1}(0)} \rightarrow E$ непреръвно, а множество значений $(f-I)\left(\overline{B_{1}(0)}\right)$ относительно компактно. Пусть существует число $\varepsilon \in(0,1)$ такое, что

$$
\|f(x)-x\| \leqslant 1-\varepsilon \quad \text { для всех } \quad x \in \partial \overline{B_{1}(0)} .
$$

Тогда справедливо включение $B_{\varepsilon}(0) \subset f\left(\overline{B_{1}(0)}\right)$.

\section{СПИСОК ЦИТИРОВАННОЙ ЛИТЕРАТУРЫ}

[1] Э. Б. Ли, Л. Маркус, Основы теории оптималъного управления, Наука, М., 1972. [2] E. S. Polovinkin, "Necessary conditions for optimization problems with differential inclusion", Set-valued Analysis and Differential Inclusions, Prog. Syst. Control Theory, 16, Birkhäuser Boston, Boston, MA, 1993, 157-170. [3] E.S. Polovinkin, "The properties of continuity and differentiation of solution sets of Lipschetzean differential inclusions", Modeling, Estimation and Control of Systems with Uncertainty, Prog. Syst. Control Theory, 10, Birkhäuser Boston, Boston, MA, 1991, 349-360. [4] Н. Данфорд, Дж. Шварц, Линейные операторы. Т. 1. Общая теория, ИЛ, М., 1962. [5] Р. Рокафеллар, Выпуклый анализ, Мир, М., 1973. [6] Е. С. Половинкин, М.В. Балашов, Элементы выпуклого и сильно выпуклого анализа, Физматлит, M., 2007.

\section{Е. С. Половинкин}

Поступило

Московский физико-технический 14.04.2014 институт (государственный университет),

E-mail: polovinkin@mail.mipt.ru 